\title{
El coste de las patologías psiquiátricas en España. Un seguimiento de 26 años y 10.974 ingresos en una Unidad Psiquiátrica de Corta Estancia de un hospital general
}

\author{
A. Seva \\ Catedrático de Psiquiatría y Psicología \\ Médica, Jefe del Departamento de \\ Psiquiatría, Facultad de Medicina y Hospital \\ Clínico Universitario, Zaragoza \\ ESPAÑA
}

\section{Introducción}

La integración de la asistencia psiquiátrica en el cuerpo asistencial del resto de la Medicina ha supuesto en el último cuarto del siglo XX un gran esfuerzo de adaptación de nuestra especialidad a las exigencias asistenciales generales impuestas por los hospitales generales, a la par que cada vez se van conociendo más y más las características propias de nuestra actividad clínicoasistencial.

A nosotros desde hace ya bastantes años nos ha preocupado el tema ya que comprendíamos que o aportábamos nosotros mismos los conocimientos que nuestros gerentes y políticos no tenían acerca de nuestra especialidad o simplemente seríamos obligados a adaptar nuestra actividad asistencial a los procederes generales de los otros servicios médicos y quirúrgicos.

En este sentido nosotros (A. Seva 1983, 1991) hace años publicamos sendas mono- grafías sobre el particular, inspiradas no solo en las experiencias internacionales más destacadas de entonces, sino básicamente en las derivadas de nuestra propia experiencia en el Departamento de Psiquiatría de nuestro Hospital Clínico Universitario en Zaragoza.

Pues bien como señala M.A. Asenjo (1999) los intereses de los personajes involucrados son diversos y así, para los pacientes lo mas importante es sentir la calidad extrín seca, concretada en que se les atienda con prontitud y amabilidad a la par que desean ser informados adecuadamente sobre las causas de su enfermedad y sobre las posibilidades terapéuticas que ellos desean asumir con responsabilidad. Pero el paciente se siente hoy también usuario de un servicio público o cliente de un servicio médico privado, con lo que exigirá que se pongan los medios personales e instrumentales del más alto nivel a su servicio. Del otro lado de la mesa del consultorio, el médico por su parte buscará ante todo la calidad científica de los 
medios puestos a su disposición para diagnosticar y tratar a su enfermo, sin que le importe demasiado el coste, la efectividad o el rendimiento de sus actuaciones. Pero para el personal que administra los recursos humanos e instrumentales, lo más importante será la eficiencia consistente en alcanzar los objetivos previamente definidos con el menor coste posible; es decir, el intentar conseguir ese dificilísimo equilibrio económico entre calidad-cantidad-coste representado por las curvas de preferencia. Estas representan las preferencias del consumidor en relación con dos variables:

1. A mayor cantidad menor coste, lo cual vendría explicado por economías de escala, curvas de experiencia y sinergias.

2. A mayor calidad mayor coste, de manera que en este caso el consumidor está menos sensibilizado con el precio del bien, buscando fundamentalmente la calidad del producto.

Pues bien, los políticos buscarán satisfacer a usuarios y a profesionales sanitarios con los limitados recursos económicos con los que cuentan, haciendo que las cuentas cuadren. Si el hospital es privado, entonces todavía más, sus dueños buscarán obtener beneficios en su actividad, y por tanto el presupuesto podría estar recortado en función de un porcentaje de beneficios exigido sin atender directamente a las necesidades presupuestarias del departamento en cuestión.

Podríamos decir que el médico es el administrador de los costes, y es por ello por lo que prácticamente la totalidad de los costes variables se explican por las decisiones de éste, no existiendo ninguna organización que controle la supervivencia del sistema. Pero es que además y por si fuera poco, los propios médicos controlamos la totalidad del conocimiento, lo que hace que el $55 \%$ de la producción científica española se refiera a temas de salud/enfermedad (Curso de Hospitales,
Escuela Nacional de Sanidad, Madrid, 1997). Por otro lado disponemos de una ética profesional, la mejor construida, a lo que se une una cultura profesional de gran peso histórico (A. Fernández Doctor 1996). Podemos así decir, que nuestro sector sanitario es el sector "más inteligente" o mejor dotado de conocimientos, contribuyendo con el $0,5 \%$ al conocimiento científico mundial.

Añadamos a todo ello, que este nuestro sector sanitario, es uno de los que más empleo genera, viviendo desde hace siglos en continuo crecimiento tecnológico, de modo que se suceden las tecnologías unas a otras antes de que se alcancen sus fases de madurez (explosión tecnológica).

El sector sanitario tiene como objetivo fundamental ofrecer un servicio a la Sociedad que a diferencia del producto acabado en una cadena de montaje, es intangible, es decir, no puede ser visto ni tocado, y además no se trata de un producto estandarizado, sino que cada servicio ofrecido es diferente al anterior y tiene en cuenta la situación personal de cada usuario (paciente). Aunque digamos que los servicios son productos intangibles, podemos encontrar componentes tangibles (fármacos, elementos ortopédicos, etc.) pero ellos no suponen el objetivo principal de la asistencia sanitaria, sino el trato con el paciente en cuestión.

En nuestro sector sanitario los compo nentes intangibles superan con creces a los tangibles, de manera que ello se nota especialmente en todo lo referente a la calidad, afectando básicamente a la satisfacción, de modo que por ejemplo,la cuarta parte de los llamados usuarios de los Servicios sanitarios, están dispuestos a pagar por que sim plemente se les escuche. La capacidad de comprensión y de comunicación, es decir, la bien conocida relación médico-enfermo, sigue siendo lo más valorado por el pacien te o el usuario, como queramos llamarle. 
Como vemos pues, no existe sector de actividad social y económica más especial que éste sanitario. Y nosotros los médicos, mediante nuestras actividades diagnósticas y terapéuticas, lo queramos admitir o no, somos los agentes generadores del gasto, moviéndonos siempre en ese difícil compromiso entre la cantidad y la calidad.

Los trabajos realizados sobre costes de las enfermedades muestran grandes variaciones en dichos costes económicos. Hace poco B.S. Bloom et al. (2001) revisaron todos los trabajos publicados entre el 1 de Enero de 1985 y el 30 de Abril de 1999 y recogidos en Medline. De un total de 1.725 trabajos solamente 110 incluían criterios de inclusión, variando extraordinariamente las medidas utilizadas.

Ya más concretamente en el caso de las patologías psiquiátricas, existen estudios recientes como el de M. Knapp et al. (1999) quienes señalan las controversias que estas investigaciones siguen planteando, no obstante lo cual defiende el hecho de que los más elevados costes estaban asociados con mejores niveles cualitativos de cuidados y con mejor calidad del Alta.

Indudablemente las Unidades de Corta Estancia de los hospitales generales han supuesto en los últimos cuarenta años un gran beneficio para nuestros pacientes. P. Johnstone y G. Zolese (1999) se referían a propósito de un estudio comparativo con los sistemas tradicionales, como los pacientes de las Unidades de Corta Estancia no generaban más reingresos, obteniéndose con ellos éxitos mejores en sus Altas. A conclusiones similares llegan autores como W.B. Hawthorme et al. (1999) quienes demuestran como los Programas de las Unidades de Corta Estancia de los hospitales generales son más económicos que los basados en la hospitalización clásica, siendo en ambos casos la patología psiquiátrica similar.
Las estancias medias de este tipo de Unidades son, además siempre menores, no superando en la mayor parte de los casos los 30 días. Los días de estancia inferiores a 30 días, guardan relación con patologías psiquiátricas menos complejas o bien con la existencia en el entorno de redes asistenciales extrahospitalarias dotadas de Programas comunitarios diversos (Hospitales de Día, Centros de Día, Atenciones domiciliarias, Hospitales de Media Estancia y de Larga Estancia, Centros de Salud Mental, etc.,etc.). Así se comprende que autores como H. Mok y C. Walter (1995) hablen de Unidades de Corta Estancia con estancias medias de tan solo 2,5 días, aunque en este caso la patología asistida era fundamentalmente trastornos de adaptación.

En algunos hospitales como el Johns Hopkins, en 1989, se introdujeron cambios organizativos con el fin de abaratar los costes del Meyer 3 Short-stay Psychiatric Service. Consistieron éstos en la utilización de criterios objetivos a aplicar por el médico a fin de que el ingreso fuese el apropiado,modificación de las visitas del doctor en las mañanas y tardes, mejora de las relaciones entre la Unidad, los Servicios de Urgencia y los Servicios comunitarios y extrahospitalarios, sustitución de los médicos residentes de primer año por residentes "senior" en los Servicios de Urgencia, debiendo además estar éstos supervisados por médicos del staff, y finalmente intensificación de las labores de enseñanza y adiestramiento dirigidas al personal de enfermería.

\section{Material y método}

Desde hace 26 años venimos coleccionando toda una serie de datos referentes a los ingresos psiquiátricos que han tenido lugar en el Servicio de Agudos del Departamento de Psiquiatría del Hospital Clínico 
Universitario de Zaragoza (España). Un total de 10.974 ingresos se realizaron entre los años 1975 al 2000. De ellos el 51 por 100 fueron hombres y el 49 por 100 mujeres. Durante la mayor parte de estos años la patología psiquiátrica aguda correspondiente a la provincia de Zaragoza (842.419 h.) fue atendida por este Servicio y a partir de 1992 y de 1994, nuevos Servicios de Agudos se abrieron en tres hospitales generales más.

Como en el año 1993 empezamos a utilizar los diagnósticos ICD-10, abandonando los ICD-9, hemos integrado a la totalidad de los diagnósticos en 22 clases con el fin de poder tratarlos como un conjunto único.

Por otro lado, hemos calculado los días de estancia producidos por cada categoría diagnóstica en estos 26 años. Teniendo en cuenta el Peso Medio (índice de complejidad de la patología) y el Coste por Unidad de Peso que en el año 2000 fue de 533.222 ptas. $(3,204.73 €)$ podemos ofrecer el Coste por cada día de estancia y el Coste al Alta.

\section{Resultados}

En la tabla I hemos representado los resultados obtenidos teniendo en cuenta los pesos específicos que miden la complejidad para cada diagnóstico psiquiátrico CIE-9 y CIE-10 integrados, las estancias medias generadas y el Coste de la Unidad de Peso. Con ello hemos calculado el valor de cada

Tabla I

Coste económico en pesetas del año 2000 de las hospitalizaciones psiquiátricas (Integración de los diagnósticos CIE-9 y CIE-10) desde el año 1975 al 2000 (26 años), Departamento de Psiquiatría (Prof. A. Seva), Hospital Clínico Universitario. Zaragoza, (n: 9.410)

\begin{tabular}{|c|c|c|c|c|c|c|c|c|}
\hline Diagnóstico & N. ${ }^{o}$ casos & $\begin{array}{c}\text { Peso } \\
\text { medio } \\
\text { (comple } \\
\text { jidad) }\end{array}$ & $\begin{array}{c}\text { Peso } \\
\text { total } \\
-(\text { comple- } \\
\text { jidad })\end{array}$ & $\begin{array}{c}\text { Coste } \\
\text { unidad } \\
\text { de } \\
\text { (peso) }\end{array}$ & $\begin{array}{c}\text { Coste } \\
\text { total } \\
\text { (euros) }\end{array}$ & $\begin{array}{l}\text { Coste } \\
\text { alta } \\
\text { (euros) }\end{array}$ & $\begin{array}{c}\text { Días } \\
\text { de } \\
\text { estancia }\end{array}$ & $\begin{array}{c}\text { Coste } \\
\text { estancia } \\
\text { (euros) }\end{array}$ \\
\hline Demencias & 219 & 2,02 & 442,38 & 533.222 & 1.417 .708 & $6.473,55$ & 26,6027 & $243,34 € €$ \\
\hline \multicolumn{9}{|l|}{ Psicosis } \\
\hline orgánicas transitorias & 138 & 1,52 & 209,76 & 533.222 & 672.224 & $4.871,19$ & 25,4275 & $191,57 €$ \\
\hline Psicosis orgánicas & 74 & 1,52 & 112,48 & 533.222 & 360.468 & $4.871,19$ & 16,5541 & $294,26 €$ \\
\hline Psicosis alcohólicas & 254 & 1,52 & 386,08 & 533.222 & 1.237 .282 & $4.871,19$ & 16,5315 & $294,66 €$ \\
\hline \multicolumn{9}{|l|}{ Dependencia } \\
\hline a drogas & 346 & $\begin{array}{c}0,795 \\
\text { (media) }\end{array}$ & 275,07 & 533.222 & 881.525 & $2.547,76$ & 13,3699 & $190,56 €$ \\
\hline Dependencia a alcohol & 450 & 0,57 & 256,50 & 533.222 & 822.013 & $1.826,7$ & 15,0289 & $121,54 €$ \\
\hline Psicosis & & & & & & & & \\
\hline esquizofrénicas & 2.153 & 1,52 & $3.272,56$ & 533.222 & 10.487 .671 & $4.871,19$ & 22,7952 & $213,70 €$ \\
\hline Trastornos paranoides & & & & & & & & \\
\hline \multicolumn{9}{|l|}{ Psicosis agudas } \\
\hline y psicógenas & 498 & 1,52 & 756,96 & 533.222 & 2.425 .852 & $4.871,19$ & 16,8414 & $289,24 € €$ \\
\hline Trastornos esquizoafectivo & os 396 & 1,52 & 601,92 & 533.222 & 1.928 .991 & $4.871,19$ & 22,5934 & $215,60 €$ \\
\hline \multicolumn{9}{|l|}{ Trastornos } \\
\hline neuróticos & 403 & $\begin{array}{c}0,9 \\
\text { (media) }\end{array}$ & 362,70 & 533.222 & 1.162 .355 & $2.884,26$ & 18,1241 & $159,14 €$ \\
\hline Trastornos de personalidac & ad 655 & 0,68 & 445,40 & 533.222 & 1.427 .387 & $2.179,22$ & 14,7191 & $148,05 €$ \\
\hline Trastornos sexuales & 3 & 1,3499 & 4,05 & 533.222 & 12.979 & $4.326,38$ & 16,6667 & $259,58 €$ \\
\hline
\end{tabular}


Tabla I (continuación)

\begin{tabular}{|c|c|c|c|c|c|c|c|c|}
\hline Diagnóstico & N. ${ }^{\circ}$ casos & $\begin{array}{c}\text { Peso } \\
\text { medio } \\
\text { (comple- } \\
\text { jidad) }\end{array}$ & $\begin{array}{c}\text { Peso } \\
\text { total } \\
-(\text { comple- } \\
\text { jidad })\end{array}$ & $\begin{array}{l}\text { Coste } \\
\text { unidad } \\
\text { de } \\
\text { (peso) }\end{array}$ & $\begin{array}{l}\text { Coste } \\
\text { total } \\
\text { (euros) }\end{array}$ & $\begin{array}{l}\text { Coste } \\
\text { alta } \\
\text { (euros) }\end{array}$ & $\begin{array}{c}\text { Días } \\
\text { de } \\
\text { estancia }\end{array}$ & $\begin{array}{c}\text { Coste } \\
\text { estancia } \\
\text { (euros) }\end{array}$ \\
\hline Disfunciones fisio & 36 & 1,3499 & 48,60 & 533.222 & 155.750 & $4.326,38$ & 17,6944 & $244,50 €$ \\
\hline Anore & 145 & 3 & 0 & 2 & 477.701 & 10.19 & 3,0000 & $308,82 €$ \\
\hline Oligofrenias & 102 & 2,02 & 206,04 & 533.222 & 660.302 & $6.473,55$ & 16,6961 & $387,72 €$ \\
\hline \multicolumn{9}{|l|}{$\begin{array}{l}\text { Trastornos Emocionales } \\
\text { y del Comportamiento }\end{array}$} \\
\hline \multicolumn{9}{|l|}{ Trastornos de Adaptación } \\
\hline & 219 & 0 & 168,63 & & 4 & 4 & 37 & $0 € €$ \\
\hline Psicosis $m c$ & 832 & 1,5 & 1.26 & & 0 & 4.8 & 23,8 & 203 \\
\hline Psicosis depresivas & 1.392 & 1,52 & $2.115,84$ & 533.222 & 6.780 .696 & $4.871,19$ & 26,1774 & $186,09 €$ \\
\hline \multicolumn{9}{|l|}{ Otras Psicosis } \\
\hline $\mathrm{Ma}$ & & & & & & & 27, & \\
\hline Distimias & 586 & 0,75 & 439,50 & 533.222 & 1.408 .479 & $2.403,55$ & 20,0222 & $120,05 €$ \\
\hline
\end{tabular}

Alta por diagnóstico psiquiátrico y el coste de cada estancia.

Las Oligofrenias con $387,72 €$, ocupan el primer lugar, seguidas de las Anorexias con 308,82 €, las Psicosis Alcohólicas con 294,66 €, las Psicosis Orgánicas con 294,26 € y las Psicosis Agudas y Psicóge - nas con $289,24 €$. Las patologías con menor coste son los Trastornos de Persona lidad, las Distimias y las Dependencias Alcohólicas.

Gráficamente aparecen representados de mayor a menor coste las patologías presentadas por nuestros pacientes (figura 1)

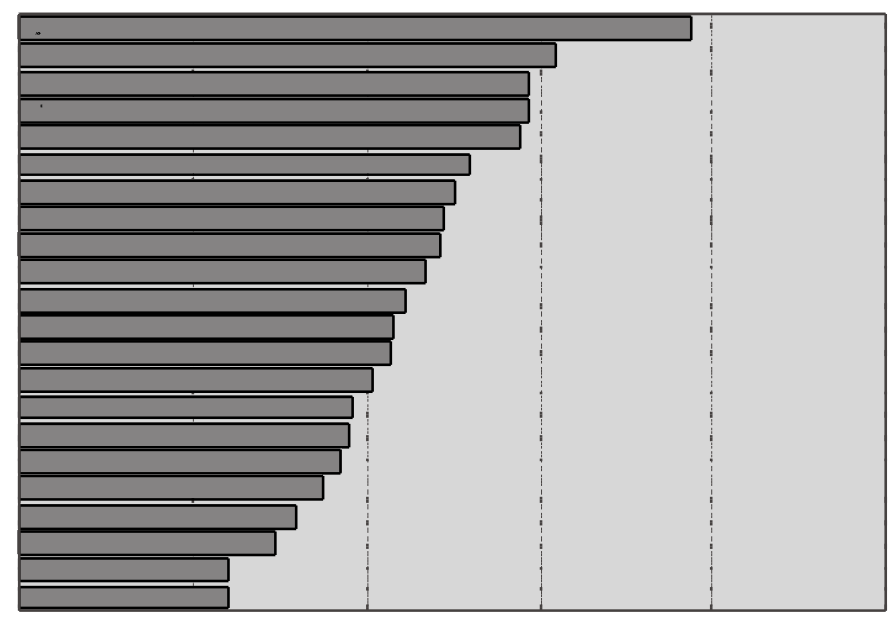

Figura 1. Coste económico en Euros del año 2000 de la estancia (Integración de los diagnósticos CIE-9 y CIE-10) teniendo en cuenta la Media de las causadas durante 26 años (1975-2000). 
En la tabla II figuran los costes que hemos calculado teniendo en cuenta el total de los días consumidos por cada diagnóstico durante estos 26 años, para lo cual introducimos en el cálculo, tanto el número de episodios de hospitalización como el número de días de cada episodio de hospitalización.

En la figura 2 podemos verlo gráficamente.

Como podemos observar las Anorexias son los procesos más caros, suponiendo un coste de 25.792,65 €, siguiéndoles los Tras tornos Esquizoafectivos con un coste en el curso de los años de 23.248,15 €. Con costes parecidos están las Otras Psicosis Maniaco depresivas 23.212,57 €. Las Psicosis Depre sivas y las Psicosis Maniacas siguen a continuación con $20.719,26 €$ y $19.466,85 €$ respectivamente. Los costes menores se dieron en Dependencias al Alcohol con 3.446,87 $€$, Trastornos de Adaptación y de Estrés con 4.017,13 €, Dependencias a Drogas con $4.270,45 €$ y Distimias con 5.422,66 €.

\section{Discusión}

Estos cálculos, como vemos están permitiendo acercarnos al conocimiento de los costes teniendo en cuenta estas variables sociodemográficas y desde luego teniendo en cuenta cada patología asistida y su variación a lo largo del tiempo; pero lo que es más importante es que facilita enormemente la comparación con el propio Servicio utilizado como patrón de referencia. Llegar a más, requeriría de estudios más profundos en los que se tuviera en cuenta, no solo los costes de las hospitalizaciones producidas por cada patología en el curso de los años, sino también los costes asistenciales de carácter ambulatorio como hace algunos años A. Seva Fernández (1996) realizó, a propósito de la esquizofrenia en Zaragoza.
A partir del estudio de una población compuesta por 111 pacientes esquizofrénicos que habían ingresado por vez primera entre los años 1976 a 1978 en nuestro Departamento de Psiquiatría, resultó que el coste de esta enfermedad en Zaragoza a lo largo de 18 años, representó en el año 1996 un montante de alrededor de veintitrés mil millones de pesetas y de treinta y tres mil millones para todo Aragón. En esta cantidad solo se incluían las hospitalizaciones, las consultas ambulatorias y los tratamientos farmacológicos, de modo que si a ello se sumaban los costes indirectos que ascendían a mil millones seiscientas mil pesetas en Zaragoza y a dos mil millones trescientas mil pesetas para todo Aragón, entonces los costes aún ascendían mucho más. Al asignar estas cantidades al año 1996,la suma de costes direc tos e indirectos alcanzaban los dos mil millones ochocientas sesenta y cinco mil pesetas, y para todo el conjunto de Aragón, ascendían a cuatro mil millones ciento setenta mil millones. A partir de ello, se pudo calcular el coste total anual de la esquizofrenia para toda España que sería de unos 70.000 millones de pesetas.

Existen amplios estudios que como el recogido en el Libro Blanco sobre el Estudio Socioeconómico sobre el Coste social de los trastornos de salud mental en España (1998) cifra en 3.373,47 € el coste de todos los trastornos mentales en nuestro país. Los costes directos representarían el 38,8\% $(1.311,69 €)$ y los indirectos el $61 \%$ $(2.061,77 €)$. Dentro de los primeros, las consultas ambulatorias suponen el 10,4\% $(352,22 €)$, los gastos de farmacia el 7,8\% $(263,50 €)$, la hospitalización el 20,6\% $(695,97 €)$, repartiéndose para la hospitali zación corta un $3 \%(98,31 €)$ y para la hos pitalización larga un 17,7\% (597,66 €). Entre los costes indirectos, la mortalidad prematura representaría el 21,6\% (730,12 $€)$, la incapacidad temporal el $8,7 \%$ 
Tabla II

Coste económico en pesetas del año 2000 de las hospitalizaciones psiquiátricas (Integración de los diagnósticos CIE-9 y CIE-10) teniendo en cuenta todo el periodo de evolución de la enfermedad durante 26 años, Departamento de Psiquiatría (Prof. A. Seva), Hospital Clínico Universitario. Zaragoza, (n: 9.410)

\begin{tabular}{lrccr} 
Diagnóstico & N. ${ }^{\text {casos }}$ & $\begin{array}{c}\text { Coste estancia } \\
\text { (euros) }\end{array}$ & $\begin{array}{c}\text { Días de estancia } \\
\text { en 26 años }\end{array}$ & $\begin{array}{r}\text { Coste proceso } \\
\text { en 26 años (euros) }\end{array}$ \\
\hline Demencias & 219 & 243,34 & 48,23 & $11.736,29 €$ \\
Psicosis orgánicas transitorias & 138 & 191,57 & 50,49 & $9.672,37 €$ \\
Psicosis orgánicas & 74 & 294,26 & 41,61 & $12.244,16 €$ \\
Psicosis alcohólicas & 254 & 294,66 & 27,92 & $8.226,91 €$ \\
Dependencia a drogas & 346 & 190,56 & 22,41 & $4.270,45 €$ \\
Dependencia a alcohol & 450 & 121,54 & 28,36 & $3.446,87 €$ \\
Psicosis esquizofrénicas & 2.153 & 213,70 & 59,16 & $12.642,49 €$ \\
Trastornos paranoides crónicos & 337 & 223,53 & 36,08 & $8.064,96 €$ \\
Psicosis agudas y psicógenas & 498 & 289,24 & 24,87 & $7.193,40 €$ \\
Trastornos esquizoafectivos & 396 & 215,60 & 107,83 & $23.248,15 €$ \\
Trastornos neuróticos & 403 & 159,14 & 38,77 & $6.169,86 €$ \\
Trastornos de personalidad & 655 & 148,05 & 41,06 & $6.078,93 €$ \\
Trastornos sexuales & 3 & 259,58 & 22,22 & $5.767,87 €$ \\
Disfunciones fisiológicas & 36 & 244,50 & 31,95 & $7.811,77 €$ \\
Anorexias & 145 & 308,82 & 83,52 & $25.792,65 €$ \\
Oligofrenias & 102 & 387,72 & 28,32 & $10.980,23 €$ \\
Trastornos emocionales & & & & \\
$\quad$ y del comportamiento Inf/Juvenil & 27 & 235,27 & 69,01 & $16.235,98 €$ \\
Trastornos de adaptación y de estrés & 219 & 251,70 & 15,96 & $4.017,13 €$ \\
Psicosis maniacas & 832 & 203,82 & 95,51 & $19.466,85 €$ \\
Psicosis depresivas & 1.392 & 186,09 & 111,34 & $20.719,26 €$ \\
Otras psicosis maniacodepresivas & 145 & 174,57 & 132,97 & $23.212,57 €$ \\
Distimias & 586 & 120,05 & 45,17 & $5.422,66 €$ \\
\hline
\end{tabular}

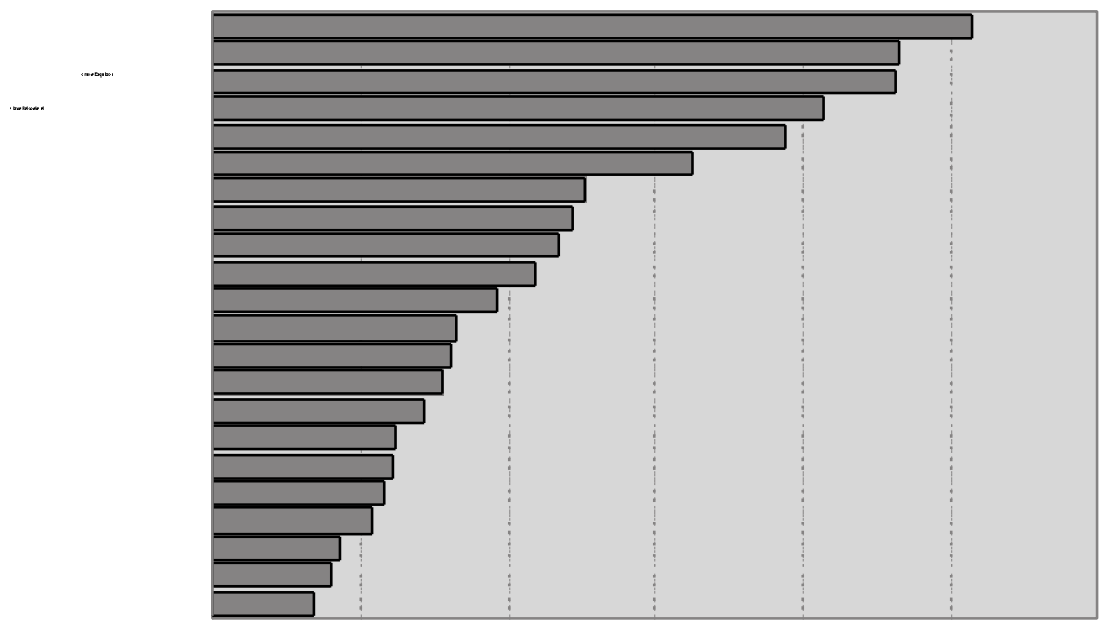

Figura 2. Coste económico en Euros del año 2000 de las hospitalizaciones psiquiátricas (Integración de los diagnósticos CIE-9 y CIE-10) teniendo en cuenta todo el periodo de la enfermedad durante 26 años (1975-2000). 
(294,50€), la invalidez el $21,8 \%$ (733,82€) y la baja productividad el $9 \%(303,33 €)$.

En cuanto a las grandes categorías diagnósticas, de acuerdo con ese estudio, podemos decir que las Psicosis (que incluirían las categorías 290 a la 299 a excepción de la 296 de la CIE-9) suponen un coste anual en España de 478,98 millones de $€$, perteneciendo a las consultas ambulatorias 45,97 millones de $€, 121,51$ millones de $€$ al gasto farmacéutico, 40,91 millones de $€$ al coste por hospitalizaciones cortas y 270,58 millones de $€$ al coste de las hospitalizaciones largas.

Las Depresiones (que incluirían a las categorías 296, 300.4 y 311 de la CIE-9) suponen un coste anual en España de 447,77 millones de $€$, de los que 187,55 millones pertenecen a las consultas ambula torias, 133,58 millones al gasto farmacéuti co, 32,42 millones de $€$ a las hospitalizacio nes cortas y 94,21 millones de $€$ a las hospitalizaciones largas.

Los Trastornos Neuróticos (categoría 300 a excepción de la 300.4) suponen un coste anual en nuestro país de 153,32 millones de $€$, repartidos en 112,23 millones de $€$ como coste de las consultas ambulatorias, 26,18 millones como gasto farmacéutico, 3,43 millones como coste de las hospitalizacio nes cortas y 11,49 millones de $€$ como coste de las largas hospitalizaciones.

Los demás trastornos mentales que son los que están incluidos en las categorías de la CIE-9 que van de la 301 a la 319 con exclusión de la 311, representan un coste anual en España de 389,60 millones de $€$, correspondiendo 48,59 millones de $€$ al coste de las consultas ambulatorias, 17,83 millones al gasto farmacéutico, 31,16 millones de $€$ al coste de las hospitalizaciones cortas $\mathrm{y}$ 292,03 millones al coste de las largas hospi talizaciones.
De acuerdo con todos estos cálculos y manejando cifras correspondientes al año 2000 , podemos decir que el coste del ingreso de una Psicosis en una Unidad de Corta Estancia sería de aproximadamente de 4.409,18 €; el de una Depresión sería de $3.938,20 €$; el de un Trastorno Neurótico sería de 2.460,81 €; y el de los demás trastornos mentales sería de aproximadamente $2.800,42 €$. En nuestro Departamento de Psiquiatría, ya vimos que la psicosis más representativa, la Esquizofrenia suponía un coste muy parecido, de $4.871,19 €$ y lo mismo podemos decir de las 4.871,19 € en el caso de las Psicosis Depresivas y los $2.403,54 €$ para las Distimias o Depresiones Neuróticas en nuestra casuística. En fin, los Trastornos Neuróticos, en nuestro caso, suponen $2.884,26 €$. En la tabla III recogemos los costes de la patología psiquiátrica, basados en los datos suministrados por el trabajo de Bernard Krief (1998) y teniendo en cuenta además nuestros propios datos.

Autores como P.J. Weiden y M. Olfson (1995) han estudiado, por ejemplo, el coste anual de las rehospitalizaciones de pacientes esquizofrénicos en los EE.UU. como consecuencia de la pérdida de eficacia de la medicación neuroléptica o del abandono de la misma. Para ello estudiaron 257.446 episodios múltiples de dos o más hospitalizaciones en pacientes esquizofrénicos que habían sido dados de alta tras una corta estancia de menos de 90 días en las Unidades Psiquiátricas de EE.UU. durante el año 1986. El coste estimado de las hospitalizaciones habidas fue de 2,3 billones de dólares del año 1993. Antes de los dos años después del Alta, el coste agregado por las rehospitalizaciones fue de aproximadamente 2 billones de dólares del año 1993. Pues bien, la pérdida de la eficacia de los neurolépticos produjo el 60 por 100 de los costes de rehospitalización y el no cumplimiento del tratamiento neuro- 
Tabla III

Costes de las enfermedades mentales en España

\begin{tabular}{|c|c|c|c|}
\hline & N. ${ }^{\circ}$ & Euros & Millones Euros \\
\hline Coste hospitalización corta Psicosis & 9.279 & $4.409,18$ & 40,91 \\
\hline Coste hospitalización larga Psicosis & 20.131 & $1.344,10$ & 270,58 \\
\hline Coste tratamiento farmacológico Psicosis & 3.364 .000 & 3,61 & 121,51 \\
\hline Coste consulta ambulatoria Psicosis & 2.110 .000 & 21,79 & 45,97 \\
\hline Coste Total & & $478,98 €$ & \\
\hline Coste hospitalización corta Depresión & 8.233 & $3.938,20$ & 32,42 \\
\hline Coste hospitalización larga Depresión & 7.009 & $1.344,10$ & 94,21 \\
\hline Coste tratamiento farmacológico Depresión & 14.067 .000 & 0,95 & 133,58 \\
\hline Coste consulta ambulatoria Depresión & 7.360 .000 & 2,55 & 187,55 \\
\hline Coste Total & & $447,77 €$ & \\
\hline Coste hospitalización corta Neurosis & 1.401 & $2.460,81$ & 3,43 \\
\hline Coste hospitalización larga Neurosis & 855 & $13.435,91$ & 11,49 \\
\hline Coste tratamiento farmacológico Neurosis & 6.580 .000 & 3,98 & 26,18 \\
\hline Coste consulta ambulatoria Neurosis & 5.130 .000 & 2,19 & 112,23 \\
\hline Coste Total & & $153,32 €$ & \\
\hline Coste hospitalización corta otro T. Mental & 11.128 & $2.800,42$ & 31,16 \\
\hline Coste hospitalización larga otro T. Mental & 21.726 & $1.344,10$ & 292,03 \\
\hline Coste tratamiento farmacológico otro T. Mental & 3.622 .000 & 4,92 & 17,83 \\
\hline Coste consulta ambulatoria otro T. Mental & 2.230 .000 & 21,79 & 48,59 \\
\hline Coste Total & & $389,60 €$ & \\
\hline
\end{tabular}

Modificada de Bernard Krief. Proyección año 2000.

léptico prescrito produjo el 40 por 100 de estos costes de rehospitalización. Mientras que lo primero sucedía sobre todo durante el primer año tras el Alta, el no cumplimiento del tratamiento se daba en el segundo año. Un aspecto igualmente importante es que los tratamientos más baratos no son necesariamente los más económicos a la larga, y ello parece ser válido tanto para los ansiolíticos (P. Martín et al., 1999) como para los neurolépticos (P.M. Galvin et al., 1999). Muy recientemente S.L. Brown (2001) estudiaba los costes de las hospitalizaciones psiquiátricas habidas en los hospitales generales de Maryland durante el año 1998 y en las que se tenía en cuenta la comorbilidad que suponía una media de siete diagnósticos secundarios.

Otros trabajos como el de A.S. Young et al. (2001) se han ocupado recientemente de todos estos problemas, aunque lo que más preocupa es como el aumento de las tasas de envejecimiento de la población y las discapacidades que conllevan, producirán un gran incremento en los costes de las enfermedades que, por ejemplo, en países como Holanda supondrá un crecimiento medio anual del 2,4 por 100 (J.J. Polder et al., 1998)

La realidad es que el enfermo psiquiátrico consume unas estancias medias bastante más altas que los demás pacientes médicos o quirúrgicos. La razón no es otra que la derivada del hecho de que, en general, nuestras enfermedades constituyen procesos largos que continuarán fuera de la Unidad del hospital general, y si falla el "apoyo familiar" ; $\operatorname{cosa}$ que desgraciadamente fallará más!, o bien la estructura asistencial comunitaria (Centros de Día, Hospitales de Día, Pisos Protegidos, Centros de Salud, etc.) no son suficientes, entonces las estancias medias bien se pro- 
longarán o simplemente aumentará la tasa de reingresos cada año.

Las explicaciones a esta situación hay que buscarlas todavía e independientemente de la idiosincrasia evolutiva de la patología psiquiátrica, en la escasez de camas de agudos en nuestros hospitales generales, en la falta de recursos asistenciales de "medias estancias" (para permanencias durante periodos de 30 a 90 días), en la falta igualmente de recursos asistenciales de "largas estancias" (de más de 90 días) y por supuesto en una carencia grande de recursos del tipo de Centros de Día, Hospitales de Día, Asistencia domiciliaria y en fin, en las deficiencias propias de la relación entre la Atención Primaria y la Especializada psiquiátrica.

Progresivamente y a partir de una mejor definición y especificación de las patologías psiquiátricas, sea mediante sistemas como los GRDs u otros, iremos construyendo medidas de eficiencia y de eficacia que nos permitan, incluso llegar a calcular con una gran exactitud los costes por proceso en Psiquiatría.

\section{Reconocimiento}

A mi hijo el Dr. D. Antonio Seva-Fernández por su inestimable ayuda durante los últimos años, en el tratamiento estadístico mediante el SPSS de la Base de Datos PONCE construida por nosotros mismos para estudios de seguimiento.

\section{Bibliografía}

ASENJO, M.A. Las Claves de la Gestión Hospitalaria, Barcelona, Ediciones Gestión 2000 S.A., 1999.
BLOOM, B.S.,BRUNO, D.J.,MAMAN, R., JAYADEVAPPA, R. Usefulness of US cost-of-illness studies in healthcare decision making. PharmacoEconomics, 19, 2: 207-213, 2001.

BROWN, S.L. Variations in Utilization and Cost of Inpatient Psychiatric Services Among Adults in Maryland. Psychiatric Services, 52, 6: 765-768, 2001.

Curso de Hospitales. Escuela Nacional de Sanidad. Madrid, 1997.

El coste social de los trastornos de salud en España. Libro Blanco Estudio Socioeconómico. Madrid, Smithkline Beecham Pharmaceuticals, 1998.

FERNÁNDEZ-DOCTOR, A. Documentos para la his toria de las profesiones médicas. El Colegio de Médicos y Cirujanos de Zaragoza (Siglos XV-XVIII), Zaragoza,Colegio Oficial de Médicos de Zaragoza, 1996.

GALVIN, P.M.,KNEZEK,L.D., RUSH,A.J., TOPRAC, M.G., JOHNSON, B. Clinical and Economic Impact of Newer Versus Older Antipsychotic Medications in a Community Mental Health Center. Clinical Therapeutics, 21, 6: 1105-1116,1999.

HAWTHORNE, W.B., GREEN, E.E., LOHR, J.B., HOUGH, R., SMITH, P.G. Comparison of Outcomes of Acute Care in Short-term Residential Treatment and Psychiatric Hospital Settings. Psychiatric Services, 50, 3:401406, 1999.

JOHNSTONE, P., ZOLESE, G. Systematic Review of the Effectiveness of Planned Short Hospital Stays for Mental Health Care. British Medical Journal,318, 7195:13871390, 1999.

KNAPP, M., HALLAM,A., BEECHAM, J. Costs and Outcomes of Mental Health Care Services. Any Difference Between Community and Private Care? Psycho, 25, 12: 736-743, 1999.

MARTIN, P. Economic Burden of Anxiety Disorders: A Review. L'Encephale, 24, 3: 280-296, 1998.

MOK, H., WATLER, C. Brief Psychiatric Hospitalisation: Preliminary Experience with an Urban Short-stay Unit. Canadian Journal of Psychiatry, 40, 7: 415-417, 1995.

POLDER, J.J., MEERDING, W.J., KOOPMANSCHAP, L., BONNEUX, L., VAN DER MAAS, P.J. The Costs of Health Care in the Netherlands in 1994: The Main Determinants were Advanced Age and Disabling Conditions. NederlandsTijdschrift voor Geneeskunde, 142, 28: 1607-1611, 1998.

SEVA,A. Ensayo para una epidemiología psiquiátrica desde la Institución Asistencial, Zaragoza, Publ. Universidad de Zaragoza, 1983. 
SEVA,A. Evaluación y estándares en la asistencia psi quiátrica, Zaragoza, Colección El Cierzo, 1991.

SEVA-FERNÁNDEZ, A. El pronóstico, la adaptación social y la calidad de vida del esquizofrénico. Estudio tras 15-18 años. Tesis Doctoral, Cátedra de Psiquiatría. Facultad de Medicina. Universidad de Zaragoza, 1996.

Taller sobre La Reforma Psiquiátrica no concluida, Sevilla, 23 y 24 de Abril, 1998.

WEIDEN, P.J., OLFSON, M. Cost of Relapse in Schizophrenia. Schizophrenia Bull., 21, 3: 419-429, 1995.
YOUNG, A.S., KAPUR, K., MURATA, D. The Time Course of Treatment Costs among Patients with Severe Mental Illness. Psychiatric Services, 52, 1: 21, 2001.

Dirección para correspondencia:

Prof. A. Seva

Catedrático de Psiquiatría

Jefe del Departamento de Psiquiatría del Hospital Clínico Universitario

c/ Domingo Miral s/n

50009 Zaragoza

ESPAÑA 